\title{
The cost-effectiveness of nivolumab monotherapy for the treatment of advanced melanoma patients in England
}

\author{
Yang Meng ${ }^{1}$ (D) Nadine Hertel $^{2} \cdot$ John Ellis $^{2} \cdot$ Edith Morais $^{2} \cdot$ Helen Johnson $^{3} \cdot$ Zoe Philips $^{1} \cdot$ Neil Roskell $^{1}$. \\ Andrew Walker ${ }^{4}$. Dawn Lee ${ }^{1}$
}

Received: 14 June 2017 / Accepted: 19 February 2018 / Published online: 9 March 2018

(c) The Author(s) 2018. This article is an open access publication

\begin{abstract}
Background Nivolumab was the first programmed death receptor 1 (PD-1) immune checkpoint inhibitor to demonstrate long-term survival benefit in a clinical trial setting for advanced melanoma patients.

Objective To evaluate the cost effectiveness of nivolumab monotherapy for the treatment of advanced melanoma patients in England.

Methods A Markov state-transition model was developed to estimate the lifetime costs and benefits of nivolumab versus ipilimumab and dacarbazine for BRAF mutation-negative patients and versus ipilimumab, dabrafenib, and vemurafenib for BRAF mutation-positive patients. Covariate-adjusted parametric curves for time to progression, pre-progression survival, and post-progression survival were fitted based on patient-level data from two trials and long-term ipilimumab survival data. Indirect treatment comparisons between nivolumab, ipilimumab, and dacarbazine were informed by these covariate-adjusted parametric curves, controlling for differences in patient characteristics. Kaplan-Meier data from the literature were digitised and used to fit progression-free and overall survival curves for dabrafenib and vemurafenib. Patient utilities and resource use data were based on trial data or the literature. Patients are assumed to receive nivolumab until there is no further clinical benefit, assumed to be the first of progressive disease, unacceptable toxicity, or 2 years of treatment.

Results Nivolumab is the most cost-effective treatment option in BRAF mutation-negative and mutation-positive patients, with incremental cost-effectiveness ratios of $£ 24,483$ and $£ 17,362$ per quality-adjusted life year, respectively. The model results are most sensitive to assumptions regarding treatment duration for nivolumab and the parameters of the fitted parametric survival curves.
\end{abstract}

Conclusions Nivolumab is a cost-effective treatment for advanced melanoma patients in England.

Keywords Nivolumab $\cdot$ Cost-effectiveness $\cdot$ Economic evaluation $\cdot$ Advanced melanoma

JEL Classification I19

Electronic supplementary material The online version of this article (https://doi.org/10.1007/s10198-018-0964-4) contains supplementary material, which is available to authorized users.

Yang Meng

ymeng@bresmed.com

1 BresMed Health Solutions, North Church House, 84 Queen Street, Sheffield S1 2DW, UK

2 Bristol-Myers Squibb Pharmaceuticals, Uxbridge, UK

3 Helen Johnson Consulting Ltd, Welwyn Garden City, UK

4 University of Glasgow, Glasgow, UK

\section{Introduction}

Melanoma is an aggressive type of skin cancer representing $4 \%$ of all UK skin cancer cases but $90 \%$ of all skin cancerrelated deaths [1]. For patients with advanced melanoma (unresectable or metastatic disease), historically prognosis has been poor, with a median survival of 6-10 months [2-6] and a 5-year survival around $10 \%[3,5,7]$.

Until recently in England, treatments available for advanced melanoma included ipilimumab and dacarbazine (mainly for patients who are ineligible for or unresponsive to ipilimumab) for patients without a BRAF mutation or BRAF wild-type (BRAF -ve). For patients with a BRAF mutation 
(BRAF +ve), ipilimumab and BRAF inhibitors (dabrafenib or vemurafenib) were standard treatments. Despite more recent therapeutic advances with ipilimumab, the long-term survival of many advanced melanoma patients remains elusive [8], with consequences felt by patients, caregivers, and society.

Ipilimumab offers potential long-term survival for 20\% of advanced melanoma patients, but not all patients respond, and there is a strong correlation between induction therapy completion and long-term survival with ipilimumab [9]. Alternatives target BRAF mutations, including dabrafenib and vemurafenib, but their clinical benefit is generally shortlived, with patients demonstrating progressive disease within 5-8 months of therapy initiation [10-12]. Importantly, BRAF mutations are only observed in approximately $50 \%$ of melanoma tumours [13]. For advanced melanoma patients who are ineligible for, or unresponsive to, ipilimumab or BRAF inhibitors, there were no alternative treatment options outside of palliative chemotherapy such as dacarbazine until recently in England.

Nivolumab is a fully human, monoclonal immunoglobulin G4 antibody that acts as a programmed death receptor 1 (PD-1) checkpoint inhibitor; blocking the interaction of PD-1 with its ligands PD-L1 and PD-L2 [14]. The mechanism of action for nivolumab is different from BRAF inhibitors. Similar to ipilimumab, nivolumab stimulates the patient's own immune system to directly fight cancer cells (as it would any other 'foreign' cell), resulting in the destruction of the tumour through pre-existing, intrinsic processes. The specific mechanism of action of immuno-oncology therapy has important implications for the duration of treatment required and the long-term survival profile expected. Nivolumab and ipilimumab act via distinct pathways, but both elicit immune memory, and thereby potentially promote long-term survival.

Based on efficacy and safety results from clinical studies including the pivotal Phase III randomised controlled trial (RCT), CheckMate 066, nivolumab monotherapy was approved by the European Medicines Agency (EMA) for a marketing authorisation in June 2015 for the treatment of advanced (unresectable or metastatic) melanoma. The National Institute for Health and Care Excellence (NICE) subsequently appraised nivolumab within its licensed indication, and a positive recommendation was issued in February 2016 [15]. This article is based on the economic analysis performed for the NICE appraisal.

Pembrolizumab, another PD-1 checkpoint inhibitor, was not considered a relevant comparator in the analysis because of the overlap in timing of the two appraisal processes; nivolumab was not a comparator in the pembrolizumab appraisal $[16,17]$.

Thus, the overall objective of the analysis was to evaluate the cost-effectiveness of nivolumab monotherapy for the treatment of advanced melanoma patients in England. BRAF mutation status was used to define two groups of patients, with ipilimumab and dacarbazine as the comparators for the BRAF - ve subgroup, and ipilimumab, dabrafenib, and vemurafenib as the comparators for the BRAF + ve subgroup; this division also allowed patient characteristics to vary between groups. The analyses for BRAF - ve and BRAF + ve patient subgroups are performed separately.

In performing such an analysis, apart from the common challenges for developing oncology cost-effectiveness models (e.g., choosing the method for modelling survival, selecting the most appropriate parametric survival curves, and extrapolating survival based on a relatively short trial period), there are additional challenges specific to modelling immunotherapy. These include modelling a group of patients who may have longer overall survival (OS) when treated with immunotherapy, but without long-term data for the new immunotherapy treatment (i.e., nivolumab). Another challenge specific to immunotherapy is 'pseudoprogression', where patients who ultimately have good OS have tumours that enlarge when assessed in the early stages of treatment, meeting the criteria for 'progressed disease' as assessed by the Response Evaluation Criteria In Solid Tumours (RECIST) criteria. This creates issues regarding the use of progression-free survival (PFS) for modelling survival, utilities, and resource use for immunotherapies.

\section{Methods}

\section{Overview}

A semi-Markov state-transition model was developed, where health-states were defined by four different measures relevant to the evaluation of the clinical and cost-effectiveness of nivolumab (see Fig. 1 for a simplified model structure):

- Progression status for modelling survival and utility: progression free, progressed, and dead.

- Time to death for modelling utility: $\geq 30$ days before death and $<30$ days before death.

- Time since treatment initiation and time to death for modelling resource use: first/second/third year after treatment initiation and 12 weeks before death (palliative care); and death.

- Treatment status for modelling nivolumab drug cost: on treatment and off treatment.

Utilities were modelled based on both progression status and time to death ( $\geq 30$ and $<30$ days before death). The standard progression-based utility approach has the limitation of not being able to capture the decline of cancer patient utilities towards the end of life. The previous studies have quantified the independent impact of time to death on cancer patient utilities, which were found to decline towards 
Fig. 1 Economic model structure
Survival
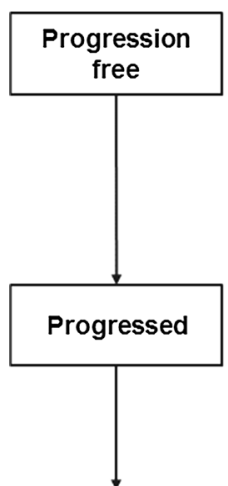

Utility

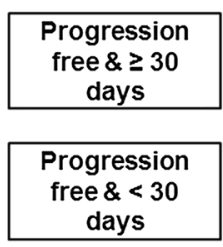

\section{Progressed}

$\& \geq 30$ days

\section{Progressed $\&<30$ days}

Resource use

Drug cost

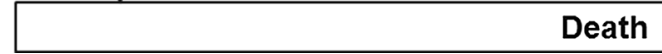

death [18-20]. Another reason for choosing a more complex method for modelling utility is the pseudo-progression experienced by some patients treated with immunotherapies (e.g., nivolumab and ipilimumab). The use of time to death utilities can mitigate the uncertainties of modelling utilities only based on progression status.

The rationale for using time since treatment initiation and time to death for modelling resource use is similar for utility modelling in that it better captures the resource use for advanced melanoma patients, given the long OS experienced and expected for these patients with immunotherapies, including nivolumab and ipilimumab [9].

The same model structure is applied to both BRAF - ve and BRAF + ve patients. The methods used for the comparison between nivolumab, ipilimumab, and dacarbazine for the BRAF - ve patient population are presented in the remainder of this section. The comparison of nivolumab with BRAF inhibitors (dabrafenib and vemurafenib) for the BRAF +ve patient population is presented in Supplemental Materials.

Key aspects of the modelling methods, assumptions, and inputs were validated by UK health economics and clinical experts.

\section{Modelling survival and safety}

There are two main approaches to implementing a three-state model: an area-under-the-curve (AUC) partitioned survival approach and a state-transition approach. The AUC approach directly estimates proportions of progression-free, progressed, and dead patients based on the OS and PFS curves. Under the state-transition approach, transition probabilities are used to estimate the proportions of progression-free, progressed, and dead patients over time.

In this analysis, the state-transition approach was used for nivolumab, ipilimumab, and dacarbazine by fitting covariate-adjusted parametric curves based on patient-level data for time to progression (TTP), pre-progression survival (PrePS), and post-progression survival (PPS). These curves were then used to derive time-dependent transition probabilities. For the BRAF inhibitors, the AUC approach was used by directly fitting OS and PFS curves using digitised Kaplan-Meier (KM) data published in the literature (see Supplemental Materials for details). In both cases, long-term survival data from non-RCT sources were also used for modelling survival beyond the trial period.

\section{Indirect treatment comparison for nivolumab, ipilimumab, and dacarbazine}

Nivolumab, ipilimumab, and dacarbazine have not been investigated together in a single clinical trial; however, patient-level data are available from two Phase III RCTs for advanced melanoma patients. The CheckMate 066 trial is a Phase III RCT for nivolumab monotherapy using dacarbazine as the control [21]. MDX010-020 is a Phase III RCT for ipilimumab and ipilimumab + gp100 using gp100 as the control [22]. The comparative efficacy of nivolumab and ipilimumab was established via a common comparator of pooled dacarbazine and gp100 and assuming the same efficacy between the two treatments, which is supported by the literature [2, 23, 24] and decision by NICE for ipilimumab [25]. To enlarge the sample size, patients in the ipilimumab and ipilimumab + gp100 arms in MDX010-020 were pooled to represent ipilimumab monotherapy, which is also supported by the trial results [26].

The indirect treatment comparison between nivolumab, ipilimumab, and dacarbazine was implemented by fitting covariate-adjusted parametric curves for TTP, PrePS, and PPS using patient-level data from CheckMate 066 and MDX010-020. The covariate-adjusted statistical model 
ensures that the differences of the covariates between the two trials and between different arms in each trial are accounted for when estimating the ceteris paribus comparative treatment effects for nivolumab, ipilimumab, and dacarbazine. Covariates were selected based on the literature and clinical relevance and include gender, age band, lactate dehydrogenase level, the Eastern Cooperative Oncology Group (ECOG) performance status, distant metastasis state, history of brain metastasis, and the use of ipilimumab as subsequent therapy. Finally, the fixed trial effect (CheckMate 066 vs MDX010-020) was included as a covariate that preserves trial randomisation and, thus, the validity of the indirect comparison between nivolumab and ipilimumab. The distribution of the covariates in different treatment arms and trials is presented in Supplemental Materials, and shows that these patient characteristics are comparable between treatment arms within a trial but less comparable between trials. It also shows that there is good overlap of covariates among different treatment arms, which is required for fitting the covariate-adjusted statistical models.

The patient characteristics used in the model were based on mean values from CheckMate 066 for BRAF -ve patients and the vemurafenib arm of the BRIM- 3 trial for BRAF +ve patients (see Table 1). The differences in patient characteristics between BRAF - ve and BRAF +ve patients reflect the different prognostic factors seen in clinical practice.

The selection of the base case parametric curves for TTP, PrePS, and PPS was based on the guidance proposed by the NICE Decision Support Unit [27]. Gompertz and loglogistic curves were selected as the base case for TTP (post100 days) and PPS, respectively, and are presented in Supplemental Materials. For the first 100 days of TTP and for PrePS, none of the parametric curves has a good visual fit; therefore, KM data were used directly and adjusted by the hazard ratios for each of the covariates estimated based on a Cox proportional hazards model (see Supplemental Materials for the detailed adjustment method and the models). The final base case TTP, PrePS, and PPS curves are also presented in Supplemental Materials.

\section{Modelling long-term survival}

Three sources of evidence were used to model long-term survival. First, for nivolumab and ipilimumab, the pooled ipilimumab long-term OS [9] was used in the model from Year 3 onwards. The ipilimumab pooled analysis is the only long-term OS evidence for immunotherapies for advanced melanoma patients, which showed a plateau in the OS beginning around Year 3, with follow-up to 10 years. The long-term ipilimumab OS is also assumed to be applicable to nivolumab because of similarity of the mechanism of action (both are immunotherapies), supported by clinical expert opinion. Second, registry OS for Stage IV melanoma reported by the American Joint Committee on Cancer (AJCC) [28], which has a follow-up period of 15 years, was used for the OS for the dacarbazine and BRAF inhibitor arms from Year 2 onwards. Finally, general population mortality based on the latest life table in England was used as the background mortality in the model [29].

Published data from the pooled ipilimumab analysis and the AJCC registry were digitised and rebased at 3 and 2 years to fit different types of parametric curves, where Gompertz and log-normal curves were chosen, respectively, for the base case based on statistical fit and clinical plausibility.
Table 1 Patient's characteristics used in the economic model

\begin{tabular}{|c|c|c|c|}
\hline & $\begin{array}{l}\text { BRAF mutation- } \\
\text { negative }^{\mathrm{a}}\end{array}$ & $\begin{array}{l}\text { BRAF mutation- } \\
\text { positive }^{\mathrm{b}}\end{array}$ & Use in the model \\
\hline Mean age & 63 & 56 & Starting age in the model \\
\hline$\%$ male & $58.9 \%$ & $59.0 \%$ & TTP, PPS, PrePS, TOT \\
\hline$\%$ under 65 & $47.8 \%$ & $100 \%$ & TTP, PPS, PrePS, TOT \\
\hline Mean weight (kg) & 78.7 & $78.7^{\mathrm{c}}$ & Drug dosing \\
\hline Mean body surface $\left(\mathrm{m}^{2}\right)$ & 1.9 & $1.9^{\mathrm{c}}$ & Drug dosing \\
\hline$\%$ stage M1c & $61.0 \%$ & $66.0 \%$ & TTP, PPS, PrePS, TOT \\
\hline ECOG status $=0$ & $64.5 \%$ & $68.0 \%$ & TTP, PPS, PrePS, TOT \\
\hline$\%$ elevated LDH $(>$ ULN) & $36.6 \%$ & $58.0 \%$ & TTP, PPS, PrePS, TOT \\
\hline$\%$ with brain metastases & $3.6 \%$ & $0 \%$ & TTP, PPS, PrePS, TOT \\
\hline$\%$ subsequent ipilimumab treatment & $29.7 \%$ & $22.0 \%$ & PPS \\
\hline
\end{tabular}

ECOG Eastern Cooperative Oncology Group, $k g$ kilogram, $L D H$ lactate dehydrogenase, $m$ metre, PPS post-progression survival, PrePS pre-progression survival, TOT time on treatment, TTP time to progression, $U L N$ upper limit of the normal range

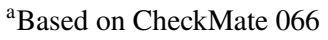

${ }^{\mathrm{b}}$ Based on BRIM-3

${ }^{\mathrm{c}}$ Assumed the same as BRAF mutation-negative patients in the absence of data 
Figure 2 presents the modelled OS for the entire time horizon based on PrePS, TTP, PPS, and long-term survival data for nivolumab, ipilimumab, and dacarbazine for BRAF - ve patients.

\section{Safety}

Patient-level adverse event (AE) data from CheckMate 066 were used to calculate the proportion of patients in the nivolumab and dacarbazine arms that experience drugrelated endocrine disorders (any grade), diarrhoea (Grade $2+$ ) and other AEs (Grade $3+$ ), with no restriction on the minimum proportion of patients experiencing an AE. The recorded hospitalisation (measured by hospital bed days) used for treating AEs and the proportion of patients requiring outpatient visits were derived from the patient-level data and the literature [30] for the costing of AEs.

For ipilimumab, dabrafenib, and vemurafenib, where patient-level AE data were not available, CheckMate 067, BREAK-3, and BRIM-3 trial data were used to derive the comparable safety model inputs relative to nivolumab and dacarbazine.

\section{Health-related quality of life}

To avoid total dependence of utility modelling on progression status and to account for the decline of utility with regard to time to death [18], a more complex method that captures both progression status and time to death was considered better suited for modelling utility in this analysis. The EQ-5D data collected in the pivotal CheckMate 066 trial were used in a longitudinal model with both progression status and whether within 30 days of death as covariates, so that utility estimates can be obtained for all four combinations of progression status and time to death (e.g., progression free and more than 30 days from death). Detailed statistical model results are presented in Supplemental Materials.

The impact of AEs on health-related quality of life was modelled by applying utility decrements, which were based on the literature [31] and applied to the percentage of patients estimated to experience each category of the modelled AEs.

\section{Time on treatment and drug costs}

Time on treatment (TOT) for nivolumab was modelled using covariate-adjusted parametric curves based on patient-level data from CheckMate 066. The method and choice of base case curve is the same as for the effectiveness endpoints, but only nivolumab data were used for fitting parametric curves (hence no treatment covariate). The log-logistic curve was chosen as the base case input based on statistical fit and clinical plausibility. For nivolumab, a maximum treatment duration of 2 years was deemed realistic in clinical practice in England by UK clinicians. Exploratory analysis from the nivolumab trials suggests that patients continue to respond long after discontinuation of treatment and that responding patients are unlikely to continue treatment with nivolumab

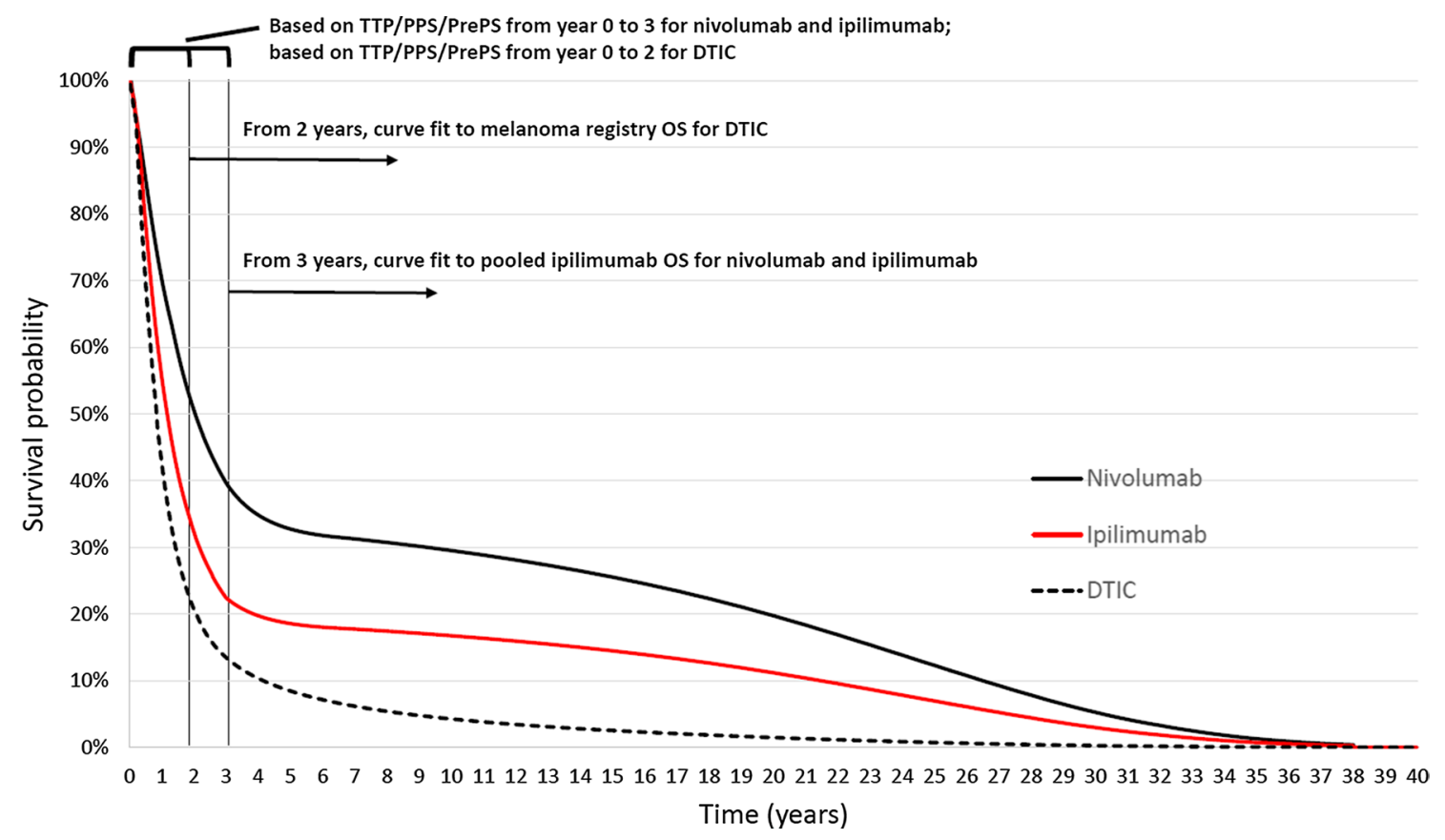

Fig. 2 Overall survival in the base case model for BRAF mutation-negative analysis. DTIC dacarbazine, OS overall survival, PPS post-progression survival, PrePS pre-progression survival, TTP time to progression 
into the long term. Scenario analyses on the length of the maximum treatment duration and on the assumed proportion of patients who discontinue treatment at 2 years were tested. The proportions of patients receiving each dose of ipilimumab were derived from the ipilimumab trials. For dacarbazine and BRAF inhibitors, it was assumed that treatment continues until disease progression.

Drug dosing for nivolumab and comparators was based on the summaries of product characteristics. Drug wastage was considered in the model. For nivolumab and ipilimumab, the dosing based on the method of moments was applied to estimate the mean number of vials required using UK patient-level weight data from nivolumab and ipilimumab trials. All drug costs were sourced from the Monthly Index of Medical Specialities (MIMS) [32] and the electronic market information tool (eMit) [33]. Ipilimumab, dabrafenib, and vemurafenib each has a separate patient access scheme (PAS), providing each drug at a discounted cost that is commercially confidential. The known ipilimumab PAS (the same manufacturer as nivolumab) and the assumed PAS for dabrafenib and vemurafenib were used in the analysis, so that the estimated cost-effectiveness results were relevant to the reimbursement decision. Drug administration costs were based on the National Health Service (NHS) reference costs [34].

\section{Resource use}

The resource use quantity and frequency were sourced from the literature $[6,25]$ and updated according to UK clinical opinion to match current treatment practice in England. The length of palliative care is assumed to be 12 weeks based on clinical opinion. Unit costs were based on NHS reference costs.

One of the covariates for the fitted TTP curves is the proportion of patients treated with ipilimumab as a subsequent line of therapy. As the subsequent use of ipilimumab affects survival, the corresponding costs were also included in the model and applied to a proportion of patients receiving ipilimumab as subsequent treatment (except in the ipilimumab arm). The mean number of ipilimumab cycles as subsequent treatment was based on a previous NICE appraisal [35].

A summary of the key base case model inputs is presented in Supplemental Materials.

\section{Sensitivity and scenario analyses}

Probabilistic sensitivity analysis (PSA) was performed to assess the uncertainties associated with key model parameters (see Supplemental Materials for detailed distributions used in PSA). Univariate one-way sensitivity analysis (OWSA) was conducted to identify the most influential parameters. Finally, a wide range of scenario analyses were used to explore the uncertainty of the model results with regard to alternative model assumptions and sources of model inputs, including alternative parametric curves and assumptions regarding the maximum duration for nivolumab.

\section{Results}

Known (for ipilimumab) and assumed (for dabrafenib and vemurafenib) PAS discounts were included in the base case incremental cost-effectiveness results for BRAF - ve and BRAF + ve patients (see Table 2). For BRAF - ve patients, nivolumab is most effective [4.31 quality-adjusted life years (QALYs)] but also most costly ( $£ 97,898$ discounted lifetime costs) compared to dacarbazine ( $£ 25,228$ and 1.23 QALYs) and ipilimumab ( 557,158 and 2.64 QALYs). For dacarbazine, drug costs are low and the majority of the estimated costs stem from subsequent ipilimumab treatment,

Table 2 Base case incremental cost-effectiveness results for BRAF mutation-negative and BRAF mutation-positive patient populations

\begin{tabular}{|c|c|c|c|c|c|c|c|}
\hline Treatment & Total costs $(£)$ & Total LYG & Total QALYs & $\begin{array}{l}\text { Incremental } \\
\text { costs }(\mathfrak{f})\end{array}$ & Incremental LYG & $\begin{array}{l}\text { Incremental } \\
\text { QALYs }\end{array}$ & ICER (£/QALY) incremental \\
\hline \multicolumn{8}{|c|}{ BRAF mutation-negative patients } \\
\hline Dacarbazine & $£ 25,228$ & 1.74 & 1.23 & & & & \\
\hline Ipilimumab & $£ 57,158$ & 3.66 & 2.64 & $£ 31,930$ & 1.92 & 1.41 & $£ 22,589$ \\
\hline Nivolumab & $£ 97,898$ & 5.75 & 4.31 & $£ 40,740$ & 2.09 & 1.66 & $£ 24,483$ \\
\hline \multicolumn{8}{|c|}{ BRAF mutation-positive patients } \\
\hline Ipilimumab & $£ 56,621$ & 3.40 & 2.44 & & & & \\
\hline Dabrafenib & $£ 71,511$ & 2.37 & 1.69 & $£ 14,891$ & -1.03 & -0.75 & Excluded due to dominance \\
\hline Vemurafenib & $£ 74,001$ & 2.37 & 1.70 & $£ 17,380$ & -1.03 & -0.74 & Excluded due to dominance \\
\hline Nivolumab & $£ 88,228$ & 5.70 & 4.27 & $£ 14,227$ & 3.33 & 2.56 & $£ 17,362$ \\
\hline
\end{tabular}

Incremental costs, LYG and QALYs are presented versus the next non-dominated comparator

$I C E R$ incremental cost-effectiveness ratio, $L Y G$ life years gained, $Q A L Y s$ quality-adjusted life years 
monitoring/palliative care/end-of-life costs, costs for the management of AEs, and drug administration costs. The breakdown of the costs for dacarbazine is presented in the Supplemental Materials. There is no dominance or extended dominance, and the incremental cost-effectiveness ratios (ICERs) for nivolumab (against ipilimumab) and ipilimumab (against dacarbazine) are estimated to be $£ 24,483$ and $£ 22,589$ per QALY gained, respectively. Therefore, nivolumab should be considered most cost-effective for BRAF - ve patients in England if the willingness-to-pay (WTP) threshold is above $£ 24,483$; ipilimumab should be considered most cost-effective if the WTP threshold is between $£ 22,589$ and $£ 24,482$; and dacarbazine should be considered most cost-effective if the threshold is below $£ 22,589$. NICE considered nivolumab to have met the criteria for an 'end of life' treatment [15]; hence, a threshold of $£ 50,000$ per QALY is assumed to be relevant. Therefore, nivolumab is estimated to be the most cost-effective treatment for BRAF - ve patients.

For BRAF + ve patients, nivolumab is also estimated to be most effective (4.27 QALYs) but also most costly $(£ 88,228)$ compared to ipilimumab ( $£ 56,621$ and 2.44 QALYs), dabrafenib (£71,511 and 1.69 QALYs) and vemurafenib (£74,001 and 2.37 QALYs). Both dabrafenib and vemurafenib are dominated by ipilimumab due to higher costs but lower QALYs compared to ipilimumab, and are, therefore, excluded as potential cost-effective treatments. The ICER for nivolumab (against ipilimumab, the only nondominated comparator) is estimated to be $£ 17,362$; therefore, nivolumab is also the most cost-effective treatment for BRAF +ve patients.

Cost-effectiveness acceptability curves (CEACs) are presented in Fig. 3 to show the probability of each treatment being the most cost-effective under different WTP thresholds (up to $£ 100,000$ ) based on 1000 PSA runs of the base case. With a WTP of $£ 50,000$, the probability of nivolumab

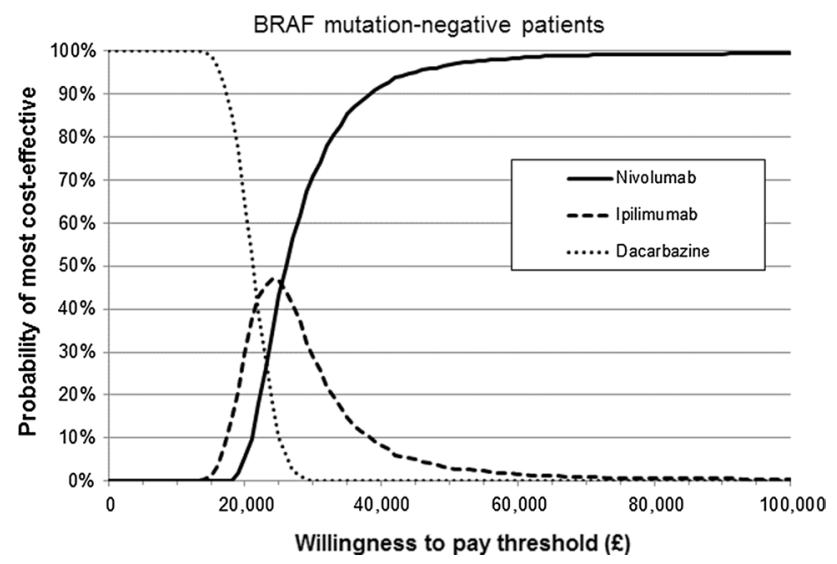

Fig. 3 Cost-effectiveness acceptability curve for BRAF mutationnegative patients being most cost-effective is 95 and $99 \%$, respectively, for the $\mathrm{BRAF}-\mathrm{ve}$ and BRAF + ve patients.

The key drivers of cost-effectiveness identified by the OWSA include covariate-adjusted survival curves for TTP and PPS for nivolumab, ipilimumab, and dacarbazine, and TOT for nivolumab; parametric curves for OS and PFS for vemurafenib and long-term OS; statistical model for deriving health-state utilities; and drug administration costs for nivolumab, ipilimumab, and dacarbazine. The OWSA results showed that the base case cost-effectiveness results are not sensitive to the most impactful parameters. The tornado diagrams, based on the OWSA, depicting the 20 most influential model inputs for BRAF -ve patients are presented in Supplemental Materials.

In the range of scenario analyses performed, nivolumab remains the most cost-effective for the majority of scenarios tested. Specifically, nivolumab remains cost-effective in scenarios for alternative parametric curves for TTP, PPS, TOT, and long-term OS; alternative data sources for indirect treatment comparison; alternative sources for utility; and alternative maximum treatment durations of 3,4 , or 5 years. Nivolumab is not cost-effective when there is a low discontinuation rate on nivolumab in Year 2 or when a high proportion of patients discontinue nivolumab treatment at Year 2 but are then assumed to have OS based on the registry data. However, these scenarios are not deemed clinically plausible based on expert opinion from UK clinicians.

\section{Discussion}

This economic evaluation was performed to help assess the cost-effectiveness of nivolumab monotherapy against all relevant comparators in England at the time of the analysis, including ipilimumab and BRAF inhibitors, by synthesising all relevant available evidence, including expert opinion. The base case ICERs estimated for nivolumab are $£ 24,482$ and $£ 17,362$ for BRAF - ve and BRAF + ve patients, respectively. These estimated ICERs are below the commonly assumed $£ 50,000$ NICE WTP threshold for end-of-life treatments. PSA estimates that the probability of nivolumab being most cost-effective compared to the comparators is 95 and $99 \%$ for BRAF - ve and BRAF + ve patients, respectively, given the WTP threshold of $£ 50,000$. Therefore, at the time of the analysis, nivolumab was considered a cost-effective treatment for advanced melanoma patients in England.

The PFS and OS estimated by the model for nivolumab and dacarbazine fit well with the pivotal CheckMate 066 trial. The longest OS data for nivolumab come from a Phase I non-RCT (CheckMate 003), with follow-up for up to 6 years [36], but patient numbers are small, with 107 advanced melanoma patients randomised to various doses 
of nivolumab and only 29, 15, and 3 at risk patients at Year 4,5 , and 6, respectively. No other long-term OS data for nivolumab are currently available. The modelled long-term OS for nivolumab lies above the OS curve for ipilimumab, which was deemed clinically plausible, given the data and evidence available and the mode of action for nivolumab. A comparison of trial observations and model predictions is presented in Supplemental Materials.

A key driver for the cost-effectiveness of nivolumab is the assumed 2-year maximum treatment period. Threshold analyses suggested that nivolumab remains cost-effective at a $£ 50,000$ WTP threshold with maximum treatment periods up to 6 and 14 years for BRAF -ve and BRAF +ve patients, respectively. It is unlikely that patients would be treated by nivolumab for such long durations in clinical practice. The optimum treatment duration for immunotherapies such as nivolumab and pembrolizumab is an active research area.

A study reported in abstract form suggests that a statetransition approach (based on TTP, PrePS, and PPS in this analysis) and an AUC approach would produce the same results [37]. The state-transition approach was chosen in the analysis, because OS data in CheckMate 066 were very immature for nivolumab, which made it difficult to estimate robust OS parametric curves for extrapolation. The state-transition approach could mitigate the limitation by fitting a more "mature" PPS compared to OS, because PPS is based on deaths for patients who have progressed, while OS is based on deaths for all randomised patients (hence, PPS has a smaller denominator). Figure 4 compares the OS and corresponding PPS KM data for the nivolumab arm in CheckMate 066 and shows that there were 50 deaths out of 210 randomised patients for the OS (median OS not reached) and 31 deaths out of 93 patients who had documented progression (median PPS is 10.9 months). The relative maturity of PPS could enable more reliable and robust extrapolation of survival beyond the trial period. The disadvantages are that PPS, compared to OS, is based on fewer data and that randomisation is not maintained for PPS as it is measured after progression.

The limitations of the study include the immature OS data from the pivotal trial, the lack of long-term OS data for nivolumab, the uncertainty regarding the treatment duration for nivolumab, and the lack of access to patient-level data for competitors' treatments. Most limitations of this analysis are common in oncology modelling, especially economic models developed to support reimbursement submissions, where there is inevitable pressure to support rapid analysis to enable access to effective and cost-effective new therapies. Therefore, in many circumstances, an economic model has to be developed with relatively immature data. However, the existence of the long-term survival data for a broadly comparable treatment, ipilimumab [9], greatly increased the robustness and credibility of long-term OS estimates for ipilimumab and nivolumab in this analysis.

When more mature nivolumab OS data from CheckMate 066 become available, it will be interesting to compare the state transition and the AUC methods using the same patient-level data. It will also be worthwhile to update the
Fig. 4 Comparison of overall survival and post-progression survival for nivolumab in CheckMate 066. $N$ total number of patients, $n$ number of patients who died, $O S$ overall survival, PPS post-progression survival

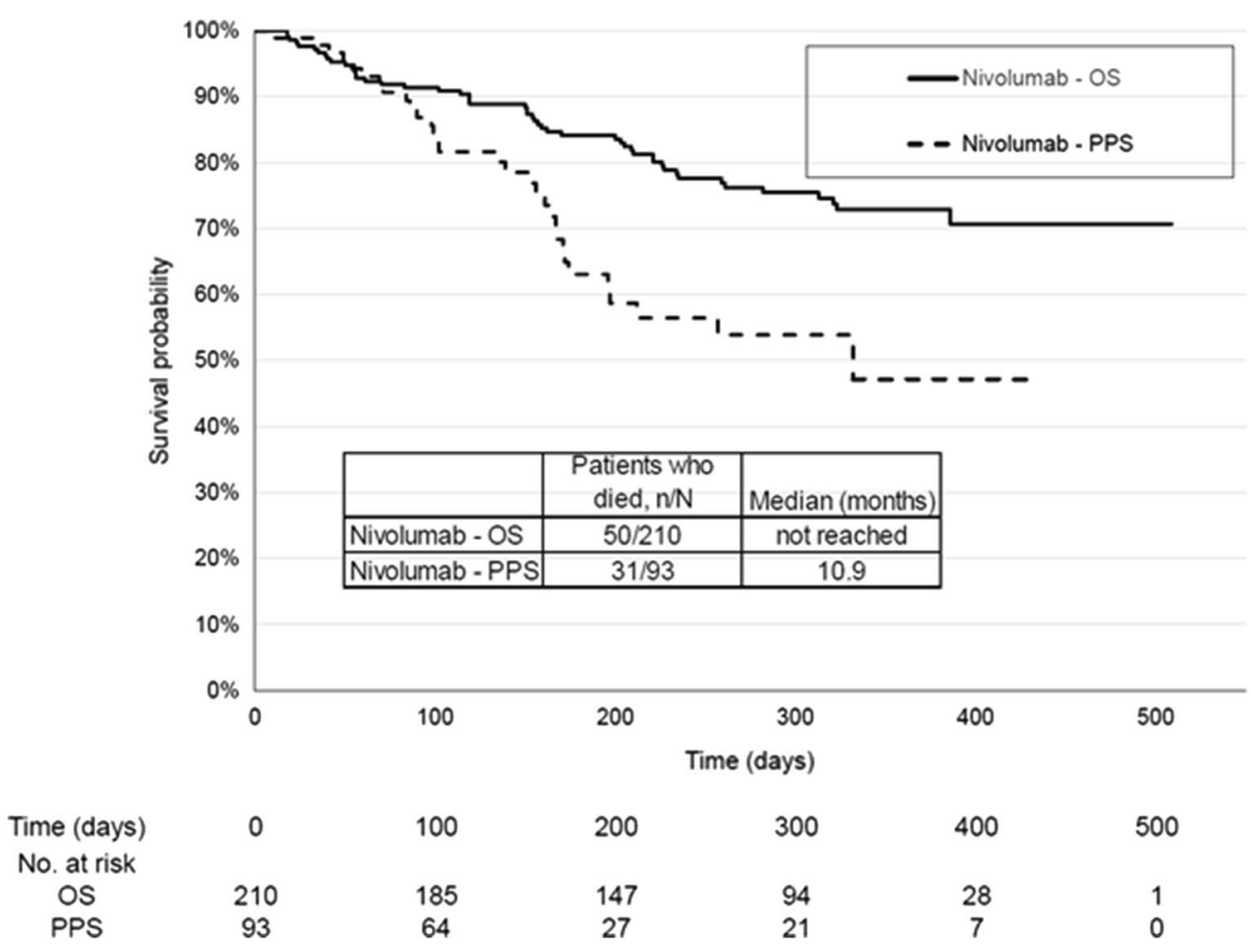


model when evidence from real-world data for optimum treatment duration for immunotherapies is available.

Since the NICE approval of nivolumab, several other new treatments (e.g., nivolumab in combination of ipilimumab and pembrolizumab) have been approved by NICE for the treatment of advanced melanoma. Therefore, future analysis would be meaningful to assess the cost-effectiveness of nivolumab monotherapy compared to these newly approved treatments which would become part of standard of care.

Alternative survival models that have the potential to model OS plateau (as estimated for the nivolumab and ipilimumab arms in this analysis) include spline models and mixture cure models. Future research on the application of these approaches will be insightful, especially when mature OS data become available.

The current analysis focuses on the cost-effectiveness of nivolumab for the treatment of advanced melanoma patients. The assessment of affordability or budget impact to the NHS in England for approving nivolumab in this indication by NICE was outside the scope of the cost-effectiveness analysis. Nevertheless, the tension between restricted health care budgets and increasing expenditures makes the general affordability of new therapies an important issue for the NHS in England and payers in other countries alike. The question of affordability may be particularly prominent for therapies, such as immune-oncology agents, that can potentially be approved in multiple indications within a short time span (e.g., nivolumab has been approved in the treatment of Hodgkin lymphoma, lung cancer, renal cell carcinoma, and head and neck cancer in England since this original analysis in advanced melanoma). It is not straightforward to assess the affordability of a particular treatment or type of treatments as this involves careful consideration of the wider health care system and other contextual factors. Further research on how the affordability issue should be assessed would be valuable to help study the affordability of new treatments.

In conclusion, current evidence indicates that nivolumab is a cost-effective treatment for advanced melanoma patients in England and represents good value for public money spent by the NHS.

Funding Financial support for this study was provided entirely by a contract with Bristol-Myers Squibb Pharmaceuticals. The funding agreement ensured the authors' independence in designing the study, interpreting the data, and writing and publishing the report

Open Access This article is distributed under the terms of the Creative Commons Attribution 4.0 International License (http://creativeco mmons.org/licenses/by/4.0/), which permits unrestricted use, distribution, and reproduction in any medium, provided you give appropriate credit to the original author(s) and the source, provide a link to the Creative Commons license, and indicate if changes were made.

\section{References}

1. Garbe, C., Peris, K., Hauschild, A., et al.: Diagnosis and treatment of melanoma. European consensus-based interdisciplinary guideline-update 2012. Eur. J. Cancer 48, 2375-2390 (2012)

2. Korn, E.L., Liu, P.Y., Lee, S.J., et al.: Meta-analysis of Phase II cooperative group trials in metastatic stage IV melanoma to determine progression-free and overall survival benchmarks for future Phase II trials. J. Clin. Oncol. 26, 527-534 (2008)

3. Balch, C.M., Gershenwald, J.E., Soong, S.J., et al.: Final version of 2009 AJCC melanoma staging and classification. J. Clin. Oncol. 27, 6199-6206 (2009)

4. Garbe, C., Eigentler, T.K., Keilholz, U., et al.: Systematic review of medical treatment in melanoma: current status and future prospects. Oncologist. 16, 5-24 (2011)

5. Agarwala, S.S.: Current systemic therapy for metastatic melanoma. Expert Rev. Anticancer Ther. 9, 587-595 (2009)

6. Lorigan, P., Marples, M., Harries, M., et al.: Treatment patterns, outcomes, and resource utilization of patients with metastatic melanoma in the U.K.: the MELODY study. Br. J. Dermatol. 170, 87-95 (2014)

7. Cancer Research UK (CRUK). Melanoma statistics and outlook. 2014

8. Kaufman, H.L., Kirkwood, J.M., Hodi, F.S., et al.: The Society for Immunotherapy of Cancer consensus statement on tumour immunotherapy for the treatment of cutaneous melanoma. Nat. Rev. Clin. Oncol. 10, 588-598 (2013)

9. Schadendorf, D., Hodi, F.S., Robert, C., et al.: Pooled analysis of long-term survival data from Phase II and Phase III trials of ipilimumab in unresectable or metastatic melanoma. J. Clin. Oncol. 33, 1889-1894 (2015)

10. Chapman, P.B., Hauschild, A., Robert, C., et al.: Improved survival with vemurafenib in melanoma with BRAF V600E mutation. N. Engl. J. Med. 364, 2507-2516 (2011)

11. Hauschild, A., Grob, J.J., Demidov, L.V., et al.: Dabrafenib in BRAF-mutated metastatic melanoma: a multicentre, open-label. Phase III randomised controlled trial. Lancet 380, 358-365 (2012)

12. Sosman, J.A., Kim, K.B., Schuchter, L., et al.: Survival in BRAF V600-mutant advanced melanoma treated with vemurafenib. N. Engl. J. Med. 366, 707-714 (2012)

13. Sullivan, R.J., Flaherty, K.T.: Resistance to BRAF-targeted therapy in melanoma. Eur. J. Cancer 49, 1297-1304 (2013)

14. Wang, C., Thudium, K.B., Han, M., et al.: In vitro characterization of the anti-PD-1 antibody nivolumab, BMS-936558, and in vivo toxicology in non-human primates. Cancer Immunol. Res. 2, 846-856 (2014)

15. National Institute for Health and Care Excellence (NICE). TA384:Nivolumab for treating advanced (unresectable or metastatic) melanoma. 2016

16. National Institute for Health and Care Excellence (NICE). TA357: Pembrolizumab for treating advanced melanoma after disease progression with ipilimumab. 2015

17. National Institute for Health and Care Excellence (NICE). TA366: Pembrolizumab for advanced melanoma not previously treated with ipilimumab. 2015

18. Hatswell, A.J., Pennington, B., Pericleous, L., et al.: Patientreported utilities in advanced or metastatic melanoma, including analysis of utilities by time to death. Health Qual Life Outcomes. 12, 140 (2014)

19. Porter J, Lee D, Hertel N, et al. Patient reported utilities in firstline advanced or metastatic melanoma: Analysis of trial CA 184024. ISPOR 17th Annual European Congress. Amsterdam: The Netherlands, 2014 
20. van den Hout, W.B., Kramer, G.W., Noordijk, E.M., et al.: Costutility analysis of short-versus long-course palliative radiotherapy in patients with non-small-cell lung cancer. J. Natl. Cancer Inst. 98, 1786-1794 (2006)

21. Robert, C., Long, G.V., Brady, B., et al.: Nivolumab in previously untreated melanoma without BRAF mutation. N. Engl. J. Med. 372, 320-330 (2015)

22. Hodi, F.S., O'Day, S.J., McDermott, D.F., et al.: Improved survival with ipilimumab in patients with metastatic melanoma. N. Engl. J. Med. 363, 711-723 (2010)

23. Kotapati S, Dequen P, Ouwens M, et al. Overall survival in the management of pretreated patients with unresectable stage III/ IV Melanoma: A systematic literature review and meta-analysis. The American Society of Clinical Oncology Annual Meeting. Chicago, IL: USA. 2011

24. Wada R, Feng Y, Zhang N, et al. Meta-analysis of Kaplan-Meier overall survival curves from selected randomized controlled Phase II/III trials in advanced melanoma. Population Approach Group Europe Meeting. Athens: Greece. 2011

25. National Institute for Health and Care Excellence (NICE). TA319: Ipilimumab for previously untreated advanced (unresectable or metastatic) melanoma. 2014

26. Larkin, J., Hatswell, A.J., Nathan, P., et al.: The predicted impact of ipilimumab usage on survival in previously treated advanced or metastatic melanoma in the UK. PLoS ONE 10, e0145524 (2015)

27. Latimer N. National Institute for Health an Clinical Excellence (NICE) DSU technical support document 14: Survival analysis for economic evaluations alongside clinical trials-Extrapolation with patient-level data. 2011
28. Balch, C.M., Buzaid, A.C., Soong, S.J., et al.: Final version of the American Joint Committee on Cancer staging system for cutaneous melanoma. J. Clin. Oncol. 19, 3635-3648 (2001)

29. Office for National Statistics (ONS). National Life Tables, England 2011-2013. 2014

30. Oxford Outcomes. Advanced melanoma resource use and costs in Europe: final report. Data on File. 2011

31. Beusterien, K.M., Szabo, S.M., Kotapati, S., et al.: Societal preference values for advanced melanoma health states in the United Kingdom and Australia. Br. J. Cancer 101, 387-389 (2009)

32. Monthly Index of Medical Specialities (MIMS). 2016

33. Department of Health. Drugs and pharmaceutical electronic market information (eMit). 2016

34. Department of Health. NHS reference costs 2014-15. 2015

35. National Institute for Health and Care Excellence (NICE). TA268: Melanoma (stage III or IV)—ipilimumab: guidance. 2012

36. Hodi S, Kluger H, Sznol M. Durable, long-term survival in previously treated patients with advanced melanoma who received nivolumab monotherapy in a phase I trial. American Association for Cancer Research (AACR) Annual Meeting. New Orleans: USA. 2016

37. Briggs, A., Baker, T., Gilloteau, I., et al.: Partitioned survival versus state transition modeling in oncology: a case study with nivolumab in advanced melanoma. Value Health J Int Soc Pharmacoecon Outcomes Res 18, A338 (2015) 